\title{
Efficacy of Virgin Coconut Oil and Chlorhexidine as an Oral Antimicrobial: A Comparative Pilot Study
}

\author{
Varsha Salian ${ }^{1}$, Damodar Hegde ${ }^{2}$, A Veena Shetty ${ }^{3}$, Mamatha Shetty ${ }^{4}$, Srikant Natarajan ${ }^{5}$
}

\begin{abstract}
Aim: The present study aimed to evaluate the efficacy of oil pulling therapy using virgin coconut oil (VCO) in reducing S. mutans counts, plaque, and gingival indices, and to compare it with the gold standard chlorhexidine.

Materials and methods: Twenty subjects (study) using VCO and 20 subjects using chlorhexidine (control) visiting the outpatient department of periodontics in the institute were chosen for the study. The gingival and plaque indices (baseline) in both groups were recorded following which unstimulated whole saliva samples were collected by spit method and sent for microbial count. They were then provided either of the VCO/mouthwash to swish once daily. Three weeks post-intervention, the recording of indices was repeated for both groups along with the microbial count.

Results: The mean values of gingival and plaque indices pre- and post-intervention showed a statistically significant reduction in the control population compared with the study group, while there was no statistically significant reduction in the bacterial count seen. The difference in the scores of plaque index pre- and post-intervention was more in control group while the difference in the gingival index was similar in both groups, but statistically insignificant.

Conclusion: Virgin coconut oil may not be as effective as chlorhexidine in reducing plaque while it may be as effective as chlorhexidine in reducing gingival index.

Clinical significance: In comparison with newer chemical oral hygiene aids, coconut oil could still be used as a traditional adjuvant to reduce gingivitis in addition to routine brushing.

Keywords: Coconut oil, Gingival index, Plaque index, Streptococcus mutans.

World Journal of Dentistry (2019): 10.5005/jp-journals-10015-1652
\end{abstract}

\section{INTRODUCTION}

Oral health is of paramount importance to all individuals. Mechanical methods of tooth cleaning are the most reliable and accepted methods for oral hygiene maintenance but adjuvants for decreasing plaque formation and maintaining oral hygiene have been always sought. ${ }^{1}$ In the oral cavity, indigenous bacteria are often associated with two major microbiological diseases, which are dental caries and periodontitis leading to pain, tooth loss, and infection. Chlorhexidine containing mouthwashes has been considered as the gold standard for the treatment of oral diseases (gingivitis and dental caries) but discouraged because of its unpleasant taste and undesirable side effects such as tooth staining. ${ }^{2}$

The traditional use of various oils in India as a means to reduce plaque formation in the oral cavity has been mentioned in the Vedic literature since ancient times. Oil pulling therapy also known as "Kavala Gandoosha" is a traditional procedure involving rinsing or swishing oil in the mouth, which is said to have anti-inflammatory and antimicrobial effect, thus reducing plaque formation in the oral cavity. Coconut oil is an edible oil that is highly desired and easily available in India and has an antimicrobial effect against a wide range of microorganisms found within the body. ${ }^{1,2}$ Lauric acid, which is a major fatty acid in coconut oil, has been very effective against viral, bacterial, fungal, and protozoal agents, which need to be compared with proven antimicrobial agents in improving oral health. Scientific evidence shows that oil pulling therapy could reduce the plaque index, and modify gingival scores and the total oral bacteria count in gingivitis patients. Virgin coconut oil obtained from the coconut kernel by wet processing is nontoxic
${ }^{1}$ Department of Oral Pathology and Microbiology, AB Shetty Memorial Institute of Dental Sciences, NITTE (Deemed to be University), Mangaluru, Karnataka, India

${ }^{2} A B$ Shetty Memorial Institute of Dental Sciences, NITTE (Deemed to be University), Mangaluru, Karnataka, India

${ }^{3}$ Department of Microbiology, KS Hegde Medical Academy, NITTE (Deemed to be University), Mangaluru, Karnataka, India

${ }^{4}$ Department of Periodontology, AB Shetty Memorial Institute of Dental Sciences, NITTE (Deemed to be University), Mangaluru, Karnataka, India ${ }^{5}$ Department of Oral Pathology and Microbiology, Manipal College of Dental Sciences, Manipal Academy of Higher Education, Mangaluru, Karnataka, India

Corresponding Author: Varsha Salian, Department of Oral Pathology and Microbiology, AB Shetty Memorial Institute of Dental Sciences, NITTE (Deemed to be University), Mangaluru, Karnataka, India, e-mail: varshavsalian@nitte.edu.in

How to cite this article: Salian V, Hegde D, Shetty AV, et al. Efficacy of Virgin Coconut Oil and Chlorhexidine as an Oral Antimicrobial: A Comparative Pilot Study. World J Dent 2019;10(4):295-300.

Source of support: Nitte (Deemed to be University) grant Rs. 10,000 for the study, which was received as a part of student research project (NUFR2)

Conflict of interest: None

and a recently emerging potent microbicidal, which is being used for oral and other purposes. ${ }^{3-5}$

Thus, the present study aimed to evaluate the efficacy of oil pulling therapy using VCO in reducing plaque and gingival indices as well as S. mutans counts in comparison with the gold standard 
chlorhexidine and thus acknowledging the use of traditional medicine as a supplemental oral hygiene aid.

\section{Materials and Methods}

On obtaining ethical clearance from institutional ethical committee, outpatients visiting the Department of Periodontics at the institution participated in this double-blinded study after prior written consent.

\section{Inclusion Criteria}

The subjects who showed their (i) willingness to participate and (ii) above 18 years of age with at least one carious tooth and moderateto-severe gingival inflammation were included.

\section{Exclusion Criteria}

(i) Subjects undergoing orthodontic treatment or using an oral prosthesis, (ii) subjects using any other mouth wash, (iii) medically compromised patients and history of recent antibiotic use were excluded.

A total of 40 individuals [20 VCO (study) and 20 chlorhexidine (control)] fulfilling the inclusion criteria were chosen. Demographic detail collection and clinical examination were performed on both groups following which gingival and plaque indices (baseline) were recorded. Unstimulated whole saliva samples were collected in

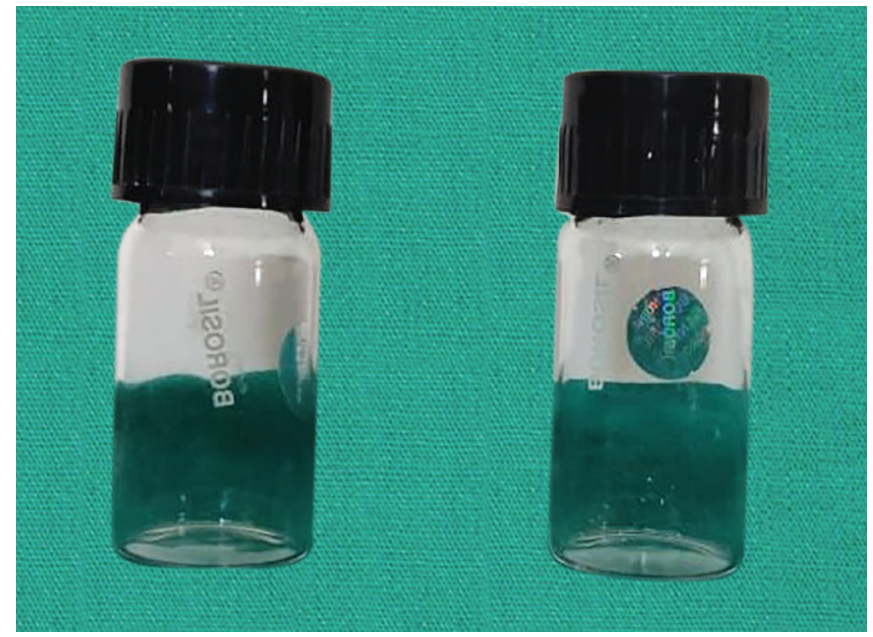

Fig. 1: Saliva collection containers

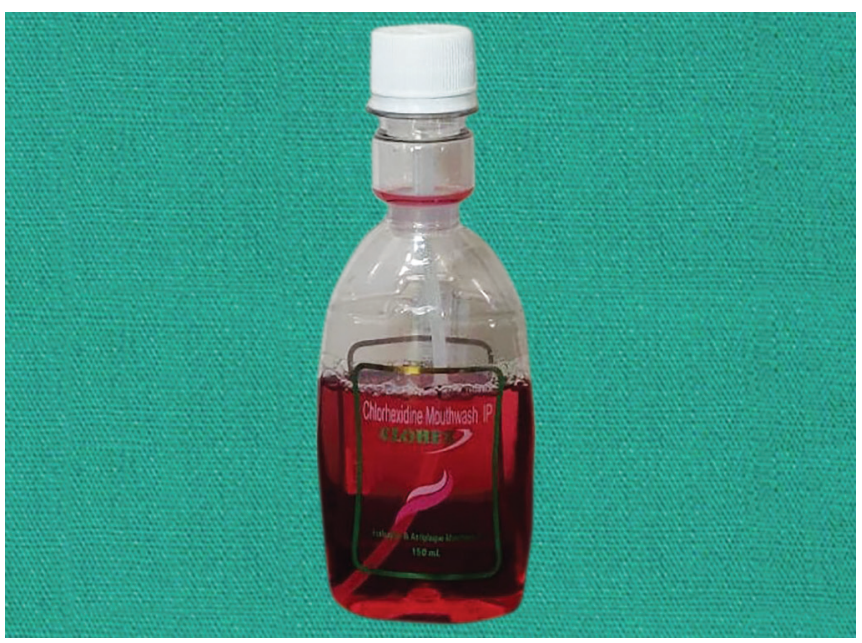

Fig. 3: Chlorhexidine mouthrinse bottle sterile saliva containers (Fig. 1) by spit method from both groups and were sent to the laboratory for the analysis of S. mutans colony counts. Following the baseline examination and saliva collection, both groups were provided with $65 \mathrm{~mL}$ of either of the VCO/mouthwash (Figs 2 and 3) in containers (Fig. 4) with instructions for usage ( $3 \mathrm{~mL}$ once a day early morning for rinsing), which was procured prior to the study. The usage of both VCO and chlorhexidine was done in addition to routine once-a-day brushing method. Three weeks post-intervention, the recording of indices and microbial counts was repeated. The gingival and plaque scores along with the microbial counts for both groups were tabulated, and results were drawn based on statistical analysis performed to compare both groups.

\section{Estimation of S. mutans Count}

The collected saliva samples of test and control subjects were streaked on to the prepared MSB Agar plates (Himedia) (Figs $5 \mathrm{~A}$ and $\mathrm{B}$ ), and the bacterial counts were recorded after 24 hours using the colony counter method of estimation.

\section{Estimation of Gingival and Plaque Indices}

The gingival and plaque indices were recorded for test and control subjects as per modified Silness and Loe criteria with index teeth chosen in every case.

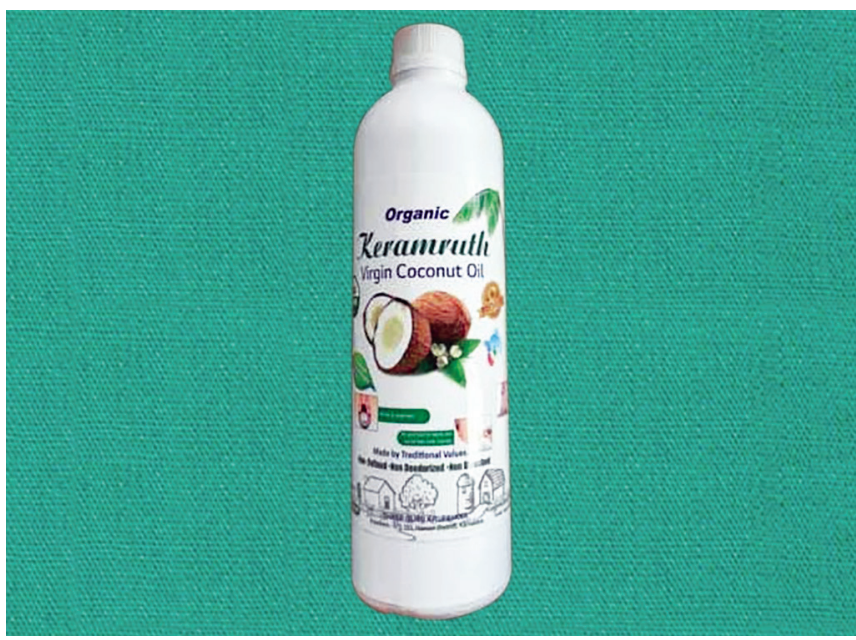

Fig. 2: Procured VCO bottle

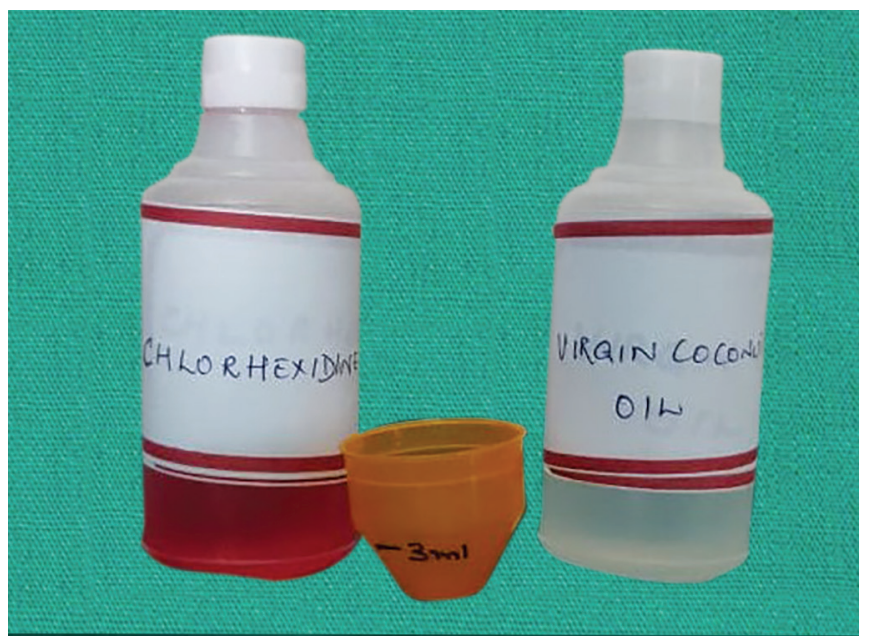

Fig. 4: Oil and mouth rinse dispensing bottles with measuring container 


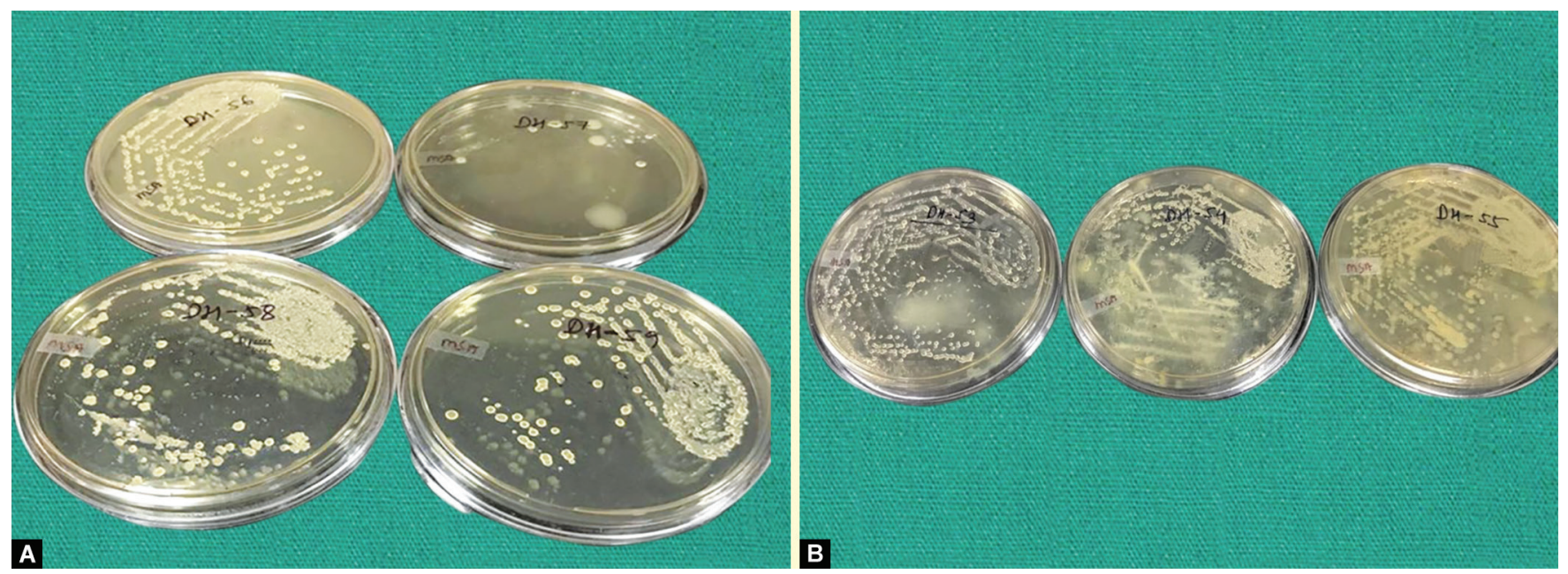

Figs 5A and B: (A) MSB agar culture plates with S. mutans colony formation in study sample; (B) MSB agar culture plates with S. mutans colony formation in control sample

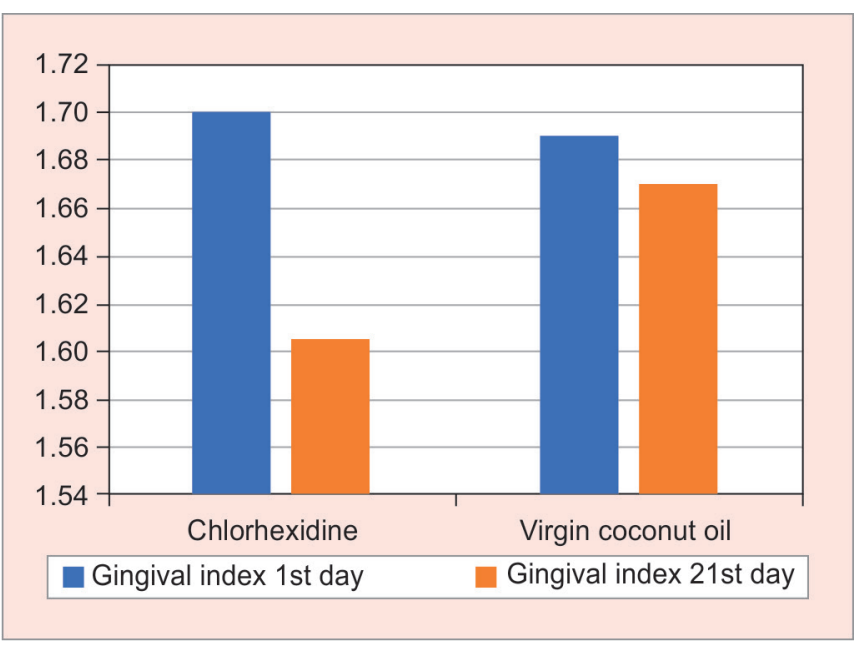

Fig. 6: Gingival indices in the study and control groups on 1st day and 21st day

The data were analyzed using SPSS 20.0 software (IBM, Chicago, IL, USA). Paired $t$ test was used to compare the gingival and plaque indices as well as bacterial load before and after the intervention. Student's $t$ test were used to compare the reduction in the gingival and plaque indices between the test and control groups. $p<0.05$ was considered as statistically significant.

\section{Results}

The mean values of gingival $(1.69,1.67)$ and plaque indices $(1.66$, $1.635)$ and bacterial counts $(5950,5050)$ pre- and post-intervention showed no statistically significant reduction in the study population. The mean values of gingival $(1.7,1.605)$ and plaque indices $(1.78,1.66)$ pre- and post-intervention showed a statistically significant reduction in the control population while there was no statistically significant reduction in the bacterial count $(5,050$, 3,700 ) seen (Table 1 and Figs 6 to 8 ). Numerically, the difference in the mean values of plaque index pre- and posttest between the two groups showed that it was significantly more in chlorhexidine

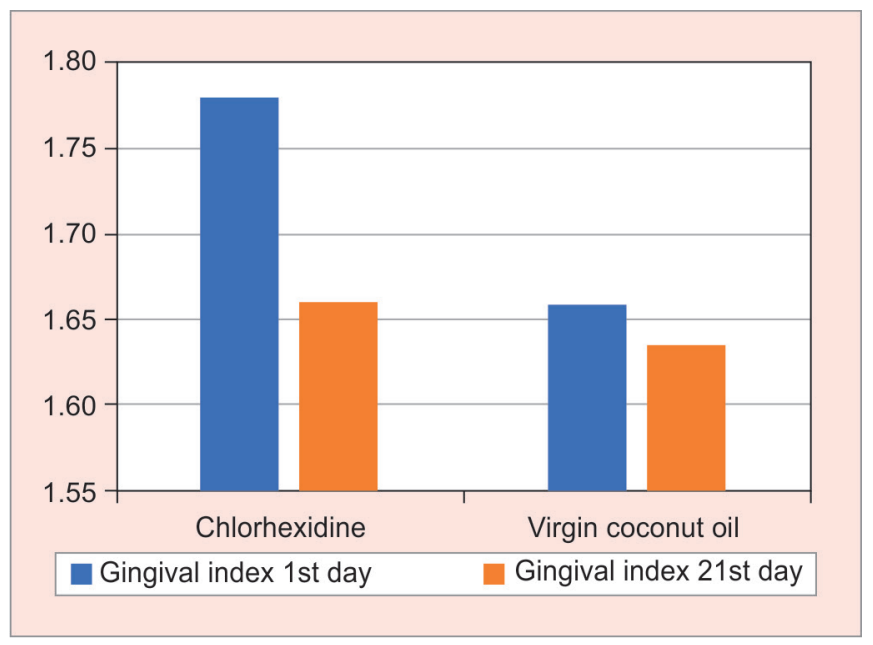

Fig. 7: Plaque indices in the study and control groups on 1st day and 21 st day

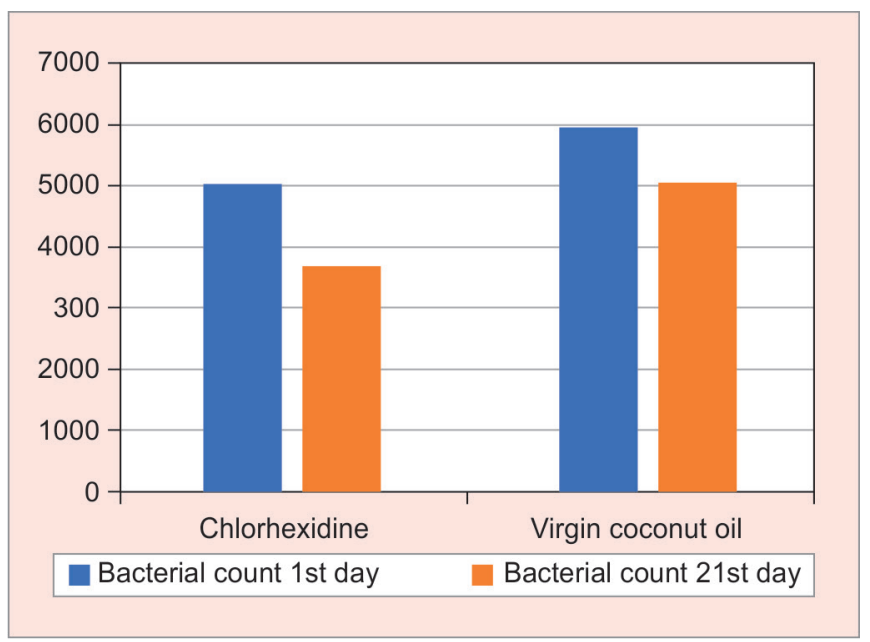

Fig. 8: Streptococcus mutans counts in the study and control groups on 1 st day and 21 st day 


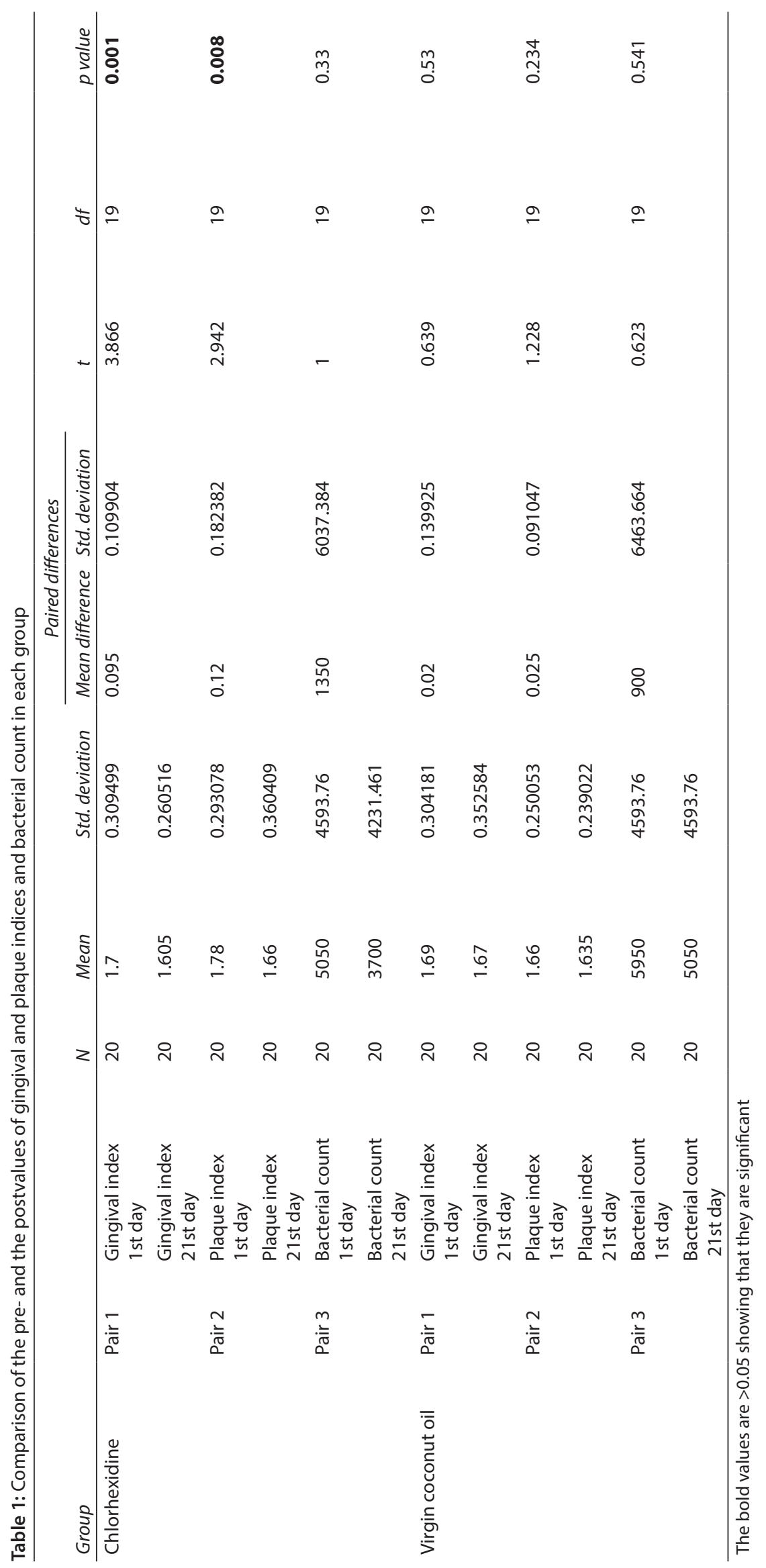


Table 2: Comparison between the two groups using independent $t$ test

\begin{tabular}{|c|c|c|c|c|c|c|c|}
\hline & Group & $N$ & Mean & Std. deviation & $t$ & $d f$ & $p$ value \\
\hline \multirow{2}{*}{$\begin{array}{l}\text { Gingival index } \\
\text { difference }\end{array}$} & Chlorhexidine & 20 & 0.095 & 0.109904 & \multirow[t]{2}{*}{1.885} & \multirow[t]{2}{*}{38} & \multirow[t]{2}{*}{0.067} \\
\hline & $\begin{array}{l}\text { Virgin coconut } \\
\text { oil }\end{array}$ & 20 & 0.02 & 0.139925 & & & \\
\hline \multirow{2}{*}{$\begin{array}{l}\text { Plaque index } \\
\text { difference }\end{array}$} & Chlorhexidine & 20 & 0.12 & 0.182382 & \multirow[t]{2}{*}{2.084} & \multirow[t]{2}{*}{27.916} & \multirow[t]{2}{*}{0.046} \\
\hline & $\begin{array}{l}\text { Virgin coconut } \\
\text { oil }\end{array}$ & 20 & 0.025 & 0.091047 & & & \\
\hline \multirow{2}{*}{$\begin{array}{l}\text { Bacterial count } \\
\text { difference }\end{array}$} & Chlorhexidine & 20 & 1350 & 6037.384 & \multirow[t]{2}{*}{0.228} & \multirow[t]{2}{*}{38} & \multirow[t]{2}{*}{0.821} \\
\hline & $\begin{array}{l}\text { Virgin coconut } \\
\text { oil }\end{array}$ & 20 & 900 & 6463.664 & & & \\
\hline
\end{tabular}

The bold values are $>0.05$ showing that they are significant

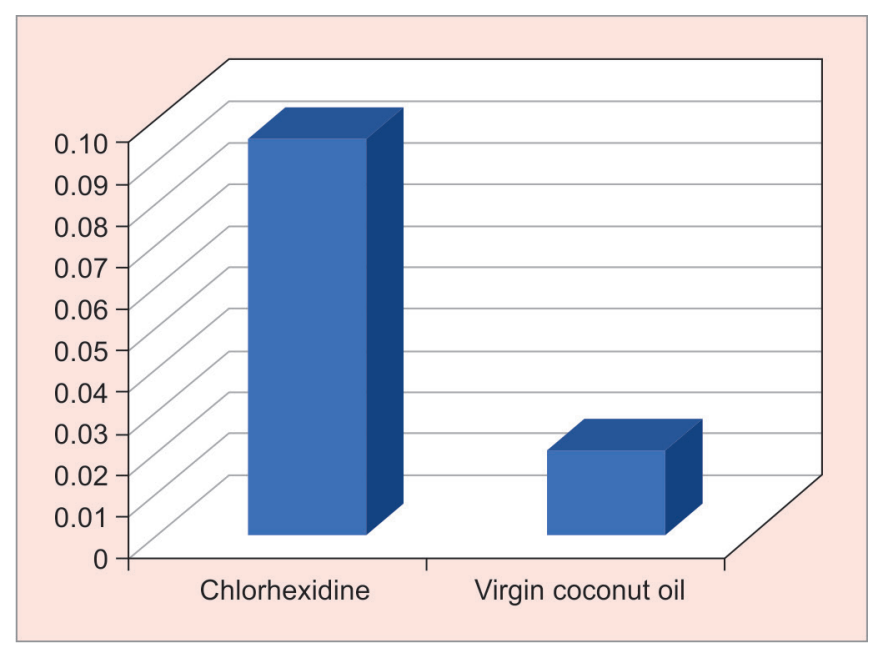

Fig. 9: Difference in the mean values of gingival indices on 1st day and 21 st day in the study and control groups

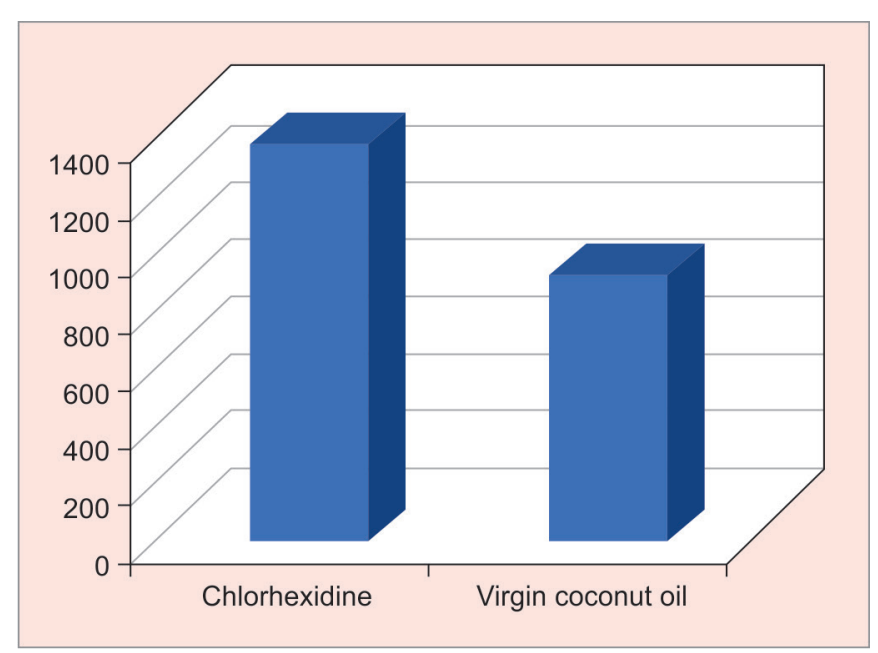

Fig. 11: Difference in the mean values of $S$. mutans counts on 1st day and 21 st day in the study and control groups

group compared with VCO group, indicating that coconut oil may not be as effective as chlorhexidine in reducing plaque. On the contrary, the difference in the mean values of gingival index preand posttest between the two groups was higher in chlorhexidine but numerically close, indicating that coconut oil may be as effective as chlorhexidine in reducing gingival index. The difference in

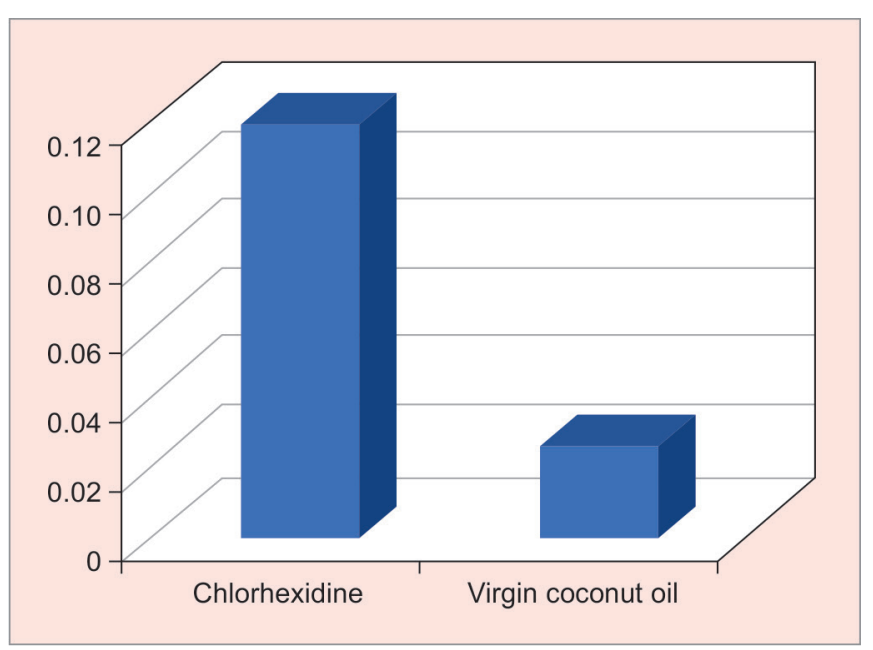

Fig. 10: Difference in the mean values of plaque indices on 1st day and 21 st day in the study and control groups

mean values of $S$. mutans counts pre- and posttest was higher in control group as compared with test group although statistically insignificant, indicating that coconut oil may not be as effective antimicrobial as chlorhexidine (Table 2 and Figs 9 to 11).

\section{Discussion}

Plaque is the primary cause of gingival inflammation, which, in turn, is the result of an interaction between plaque, tissues, and inflammatory response of the host. Chemical means of plaque control involve the use of mouth rinses, which reduce the incidence of plaque-related diseases by decreasing the plaque accumulation. ${ }^{1}$ Oil pulling or oil swishing as mentioned in our Vedic literature is an age-old traditional remedy to prevent most of the diseases involving teeth and gums and could be used for the same purpose as above. ${ }^{6}$ Our study aimed at checking the effectiveness of oil pulling with VCO as an adjuvant to brushing, in decreasing the plaque accumulation, plaque-induced gingivitis, and S. mutans counts, as compared with the gold standard, chlorhexidine. Plaque Index by Sillness and Loe and modified gingival index were used for clinical assessment as they are widely used indices. ${ }^{1,7}$ Various edible oils that have been used by authors in previous studies include sesame oil, groundnut oil, corn oil, olive oil, mustard oil, rice bran oil, palm oil, sunflower oil, soya bean oil, and coconut oil. 6,8,9 The efficacy of coconut oil as an antimicrobial and as an antiplaque agent has been tested by Ogbolu et al. Taheri et al. 
Thaweboon et al., Hughes, Singla et al., Peedikayil et al., Shino et al., and Salian et al., and the results have shown that coconut oil is effective in reducing oral microbial load and decreasing plaque and gingival indices. ${ }^{1,2,5,9-13}$ Chlorhexidine-containing mouthwashes provide a "gold standard" by which to assess the efficacy of other agents that are used as clinical adjuvants in the treatment of both caries and periodontal diseases. ${ }^{8}$ They have been extensively used as clinical adjuvants but have been recently discouraged because of their unpleasant taste and undesirable side effects such as tooth staining. Additionally, chlorhexidine might not be easily accessible and affordable to low socioeconomic group of people. ${ }^{2}$ In our study, the control population using chlorhexidine mouthwash showed a statistically significant decrease in plaque and gingival indices pre- and post-intervention, while the bacterial counts remained unchanged. However, it did not show any statistically significant reduction in plaque and gingival indices or bacterial counts in the study population performing coconut oil pulling. This could be attributed to the fact that oils do not directly inhibit microorganisms, and the fatty acid content in these oils, which are present as triglycerides, when in free form show an inhibitory action. ${ }^{12}$ Monolaurin is the monoglycerides of lauric acid, which in turn is the free fatty acid found in coconut oil. Monolaurin is shown to have antimicrobial activity against various Gram-positive and Gram-negative organisms. ${ }^{9}$ In our case, since monolaurin was not used in its free fatty acid form, the antimicrobial action of coconut oil may not have been executed to its full potential. Various studies in the past such as by Thaweboon et al., Asokan et al., Singla et al. have demonstrated oil pulling using different oils, which were shown to reduce the oral microorganisms especially S. mutans. $2,11,14$ However, the study by Jauhari et al. showed no significant reduction in the bacterial counts. ${ }^{15}$ Kaushik et al. compared the saliva samples for S. mutans count on participants using coconut oil, chlorhexidine, and distilled water for 15 days. ${ }^{16}$ A statistically significant reduction in S. mutans was seen in both study and control groups. Our study did not show any statistically significant reduction in these bacterial counts in both groups. Our study, however, showed differences in the numerical values of gingival and plaque scores as well as S. mutans counts, pretest, and posttest, in both study and control groups, although statistically insignificant. Singla et al. in their study used sesame oil, olive oil, and coconut oil along with chlorhexidine as gum massage agents and showed that there was a significant reduction in the values of S. mutans, plaque scores, and gingival scores among the four groups. ${ }^{2}$ However, the difference in percentage reduction of the measured parameters among the four groups was not statistically significant. ${ }^{2}$ Thus, this shows that there could be many more factors that may influence the action of oil pulling procedure other than the oil on its own.

\section{Limitations of the Study}

The minimum sample size required to carry out the study was calculated keeping in mind the time constrains. The taste of oil during oil pulling makes it unacceptable for certain individuals, thus making it difficult to be used for a larger sample size. The lack of significance between the groups could be due to the smaller sample size. Similarly, longer periods of follow-up may have an effect on the significance of the study. Monitoring of patients in addition to giving instructions for usage must be done, which could affect the assessment parameters in the study.

\section{Conclusion}

Considering the limitations of the present study, it can be concluded that oil pulling may not be a replacement for existing oral hygiene aids but can be an adjuvant to tooth brushing. Coconut oil pulling may not be as effective as chlorhexidine in reducing plaque index and S. mutans counts in saliva, but it seems that it may be as effective as chlorhexidine in reducing gingival index. However, oils may be valuable preventive agents in maintaining and improving oral health in the low socioeconomic group in society.

\section{References}

1. Peedikayil FC, Sreenivasan P, Narayanan A. Effect of coconut oil in plaque related gingivitis: a preliminary report. Niger Med J 2015;56(2):143-147. DOI: 10.4103/0300-1652.153406.

2. Singla N, Acharya S, Martena S, et al. Effect of oil gum massage therapy on common pathogenic oral microorganisms: a randomized controlled trial. J Indian Soc Periodontol 2014;18(4):441-446. DOI: 10.4103/0972-124X.138681.

3. DebMandal M, Mandal S. Coconut (Cocos nucifera L.: Arecaceae): In health promotion and disease prevention. Asian Pac J Trop Med 2011 Mar;4(3):241-247. DOI: 10.1016/S1995-7645(11)60078-3.

4. Marina AM, Che Man YB, Amin I. Virgin coconut oil: emerging functional food oil. Trends Food Sci Technol 2009;20:481-487. DOI: 10.1016/j.tifs.2009.06.003.

5. Ogbolu DO, Oni AA, Daini OA. In Vitro antimicrobial properties of coconut oil on Candida species in Ibadan, Nigeria. J Med Food 2007;10(2):384-387. DOI: 10.1089/jmf.2006.1209.

6. Tomar P, Hongal S, Jain M. Oil Pulling, and Oral Health: A Review. IJSS Case Report \& Reviews 2014;1(3):33-37.

7. Ciancio SG. Current status of indices of gingivitis. J Clin Periodontol 1986;13:375-378. DOI: 10.1111/j.1600-051X.1986.tb01476.x.

8. Amith HV, Ankola AV, Nagesh L. Effect of oil pulling on plaque and gingivitis. J Oral Health Community Dent 2007;1:12-18. DOI: 10.5005/ johcd-1-1-12.

9. Salian V, Shetty P. Coconut Oil, and Virgin Coconut Oil: An Insight into its Oral and Overall Health Benefits. J Clin Diagn Res 2018;12(1): ZE01-ZE03. DOI: 10.7860/JCDR/2018/31409.11051.

10. Taheri JB, Espineli FW, Lu H, et al. Antimicrobial effect of coconut flour on oral microflora: An in vitro study. Res J Biol Sci 2010;5(6):456-459. DOI: 10.3923/rjbsci.2010.456.459.

11. Thaweboon S, Nakaparksin J, Thaweboon B. Effect of Oil-Pulling on Oral Microorganisms in Biofilm Models. Asia J Public Health 2011;2(2):62-66.

12. Hughes PI, Kealey C, Rowan NJ, et al. Evaluation of vegetable oils and their respective fatty acids on the viability of Streptococcus mutans, A persistent oral Pathogen. J Asian Sci Res 2013;3(6):670-676.

13. Shino B, Peedikayil FC, Jaiprakash SR, et al. Comparison of Antimicrobial Activity of Chlorhexidine, Coconut Oil, Probiotics, and Ketoconazole on Candida albicans Isolated in Children with Early Childhood Caries: An In Vitro Study. Scientifica(Cairo) 2016;2016, 7061587. DOI: 10.1155/2016/7061587.

14. Asokan S, Rathinasamy TK, Inbamani N, et al. Mechanism of oilpulling therapy: in vitro study. Indian J Dent Res 2011;22(1):34-37. DOI: 10.4103/0970-9290.79971.

15. Jauhari D, Srivastava N, Rana V, et al. Comparative Evaluation of the effects of fluoride mouth rinse, herbal mouth rinse and oil pulling on the caries activity and Streptococcus mutans count using oratest and dentocult SM strip mutans kit. Int J Clin Pediatr Dent 2015 MayAug;8(2):114-118. DOI: 10.5005/jp-journals-10005-1295.

16. Kaushik M, Reddy P, Sharma R, et al. The Effect of Coconut Oil pulling on Streptococcus mutans count in Saliva in Comparison with Chlorhexidine Mouthwash. J Contemp Dent Pract 2016;17(1):38-41. DOI: 10.5005/jp-journals-10024-1800. 